2017-12-14

\title{
Analysing the Sentiment Expressed by Political Audiences on Twitter: The case of the 2017 UK General Election
}

Plummer, M

http://hdl.handle.net/10026.1/14112

10.1109/CSCI.2017.253

ISBN: 1-60132-469-3

IEEE

All content in PEARL is protected by copyright law. Author manuscripts are made available in accordance with publisher policies. Please cite only the published version using the details provided on the item record or document. In the absence of an open licence (e.g. Creative Commons), permissions for further reuse of content should be sought from the publisher or author. 


\title{
Analysing the Sentiment Expressed by Political Audiences on Twitter:
}

\author{
The case of the 2017 UK General Election
}

\author{
Matthew Plummer ${ }^{1}$, Marco A. Palomino ${ }^{2}$, Giovanni L. Masala ${ }^{3}$ \\ Big Data Group, School of Computing, Electronics and Mathematics \\ University of Plymouth, UK \\ ${ }^{1}$ matthew.plummer@students.plymouth.ac.uk \\ 2 marco.palomino@plymouth.ac.uk \\ ${ }^{3}$ giovanni.masala@plymouth.ac.uk
}

\begin{abstract}
A significant amount of research on the intersection of sentiment analysis and social media platforms has been published in the past few years. While previous studies have focused on methods to identify the polarity of online posts, little has been done in terms of using the impact of such posts to enhance the discovery and description of trends in real time. Here, we present a tool for the retrieval and analysis of microblogging posts in real time. We have gathered a large sample of tweets related to the 2017 UK General Election. We introduce a novel classification of the polarity of sentiments, considering the correlation between words, events and sentiments.
\end{abstract}

Keywords: Information Retrieval Methods; Social Networks; Web Search and Information Extraction; Predictive Analytics

Full/regular research papers to CSCI-ISBD

\section{INTRODUCTION}

Twitter has become the main means for microblogging, a new form of communication that allows users to create short messages and share them with an online audience [1]. Twitter is particularly fashionable on the mobile Web, where interacting with others has become more convenient, compared to the days when desktop browsing and interaction was the norm. The popularity of Twitter has risen significantly since the first tweet was published on 21 March 2006 [2]. It took longer than three years for the first one billion tweets to be published; but it currently takes less than two days to see one billion new tweets available online [3].

In the last decade, Twitter and other social media platforms have turned out to be the new venues where political campaigns and debates take place [4]. Online news portals have disseminated political stories at unprecedented rates, and politicians have got used to advertising their own messages through microblogging, which is an affordable and quick route for connecting with citizens [5].

Now that political text is at our fingertips, important research questions are beginning to emerge. There is a growing body of literature suggesting the use of social-media to forecast electoral results [5-7]. Although some scholars think that the predictive power of Twitter has been greatly exaggerated [8,9], others have been able to establish a noteworthy correlation between social media and the results of traditional mass surveys [10]. As more and more users "tweet" about their political views, Twitter has become an invaluable source of people's opinions and sentiments.
Consequently, alongside the interest in using Twitter as a predictive tool for political campaigns, the analysis of the sentiment communicated has caught the attention of computer scientists. At present, sentiment analysis - the process of determining the emotional tone behind words, used to gain an understanding of attitudes, opinions and emotions expressed within an online post-is one of the most active areas of research [11].

We do not attempt to make predictions using Twitter. However, we are interested in learning how we can use sentiment analysis to improve our understanding of political audiences. Whilst former research has classified sentiment into positive, negative and neutral categories, we have introduced a distinction between "neutral" tweets-where no sentiment is conveyed-and "balanced" tweets-where positive and negative sentiments are mixed at equal levels.

The remainder of this paper is organized as follows: Section II summarizes the relevant literature related to our work. Section III describes the methods that we employed to collect tweets and perform the data analysis. Section IV presents our results. Finally, Section V states our conclusions and outlines opportunities for future work.

\section{RELATED WORK}

Political scientists have a long history of studying the effects of campaigns and public debates [6]. However, multidisciplinary collaborations linking the fields of political science and computational text analysis have recently flourished [5]. There is an emergent body of computer science literature looking into the use of social media to analyze and predict elections [6].

Tumasjan et al. [13] have found that the number of tweets mentioning a party reflects election results, while O'Connor et al. [14] have suggested that tweets with sentiment can potentially serve as votes and substitute traditional polling. Wang et al. [6] built a random walk model with a time trend to estimate a presidential candidate's performance in public debates, assuming that strong performance in debates leads to faster follower growth. There seems to be a high parallel between performance in the polls and popularity in Twitter, as pointed out by Wang et al. [7], when researching the 2016 US Presidential Campaign.

As opposed to the related work summarized here, we do not attempt to make electoral predictions based on Twitter. Although we acknowledge previous work that shows the potential of Twitter predictive models [15], we believe that a 
number of external factors influencing the public opinion cannot be explained, exclusively, through the analysis of sentiment expressed on tweets. Additionally, there might be significant differences in the use of Twitter-and social media in general - in different countries and geographical regions. Hence, methods for predictions made for an electoral event in the US cannot be replicated anywhere else without refinements and careful consideration. In any case, our results might in turn help others to improve the forecasting power of social media. Our goal, however, is to identify the possible correlations between the sentiment expressed on Twitter and the occurrence of important events taking place during a campaign.

\section{METHODS}

In order to have a testbed to experiment with the retrieval and analysis of tweets, we have developed a Java-based application that interacts with the Twitter API [16] to retrieve public tweets. We have used the Intellij IDE [17] to program such an application. The interaction with the Twitter API is handled by Twitter $4 j$ [18], a Java library for the Twitter API. Our application uses both cloud storage and cloud computation: MongoDB Atlas [19] is employed to store the data retrieved; and MongoDB Compass [20] is employed to visualize, browse and, if needed, edit data.

MongoDB Atlas keeps all of the data retrieved from Twitter-i.e., the tweets and their associated metadatatogether with the results of the sentiment analysis process. MongoDB Atlas also keeps a list of English positive and negative opinion words, or sentiment words. We are using the list of opinion words compiled by $\mathrm{Hu}$ and Liu originally in 2004 [21]. Such a list is updated regularly for research purposes. At present, the list comprises 6,800 words, approximately. Our application analyzes tweets in real-time, resulting in real-time visualization of data. MongoDB Compass visualizes the data contained within the MongoDB Atlas cluster. This provides quick feedback related to the raw data and the sentiment analysis carried out. For the purpose of analytics, we chose Knowi [22], an online analytics tool that facilitates the combination of a variety of data sources. Thanks to Knowi, we can produce reports and visualizations at different stages of the analysis.

The core components of the proposed architecture are:

- The Twitter API

- Twitter4j

- MongoDB Atlas

- MongoDB Compass

- Knowi

When running our application for the first time, we execute a set of scripts on the MongoDB Atlas database, which populate the 'Sentiment' collection with the list of sentiment words needed to analyze the tweets.

Our application incorporates a graphical user interface (GUI) that potential users can employ to specify the necessary parameters for the retrieval of tweets. Such parameters are, for example, the maximum number of tweets to retrieve; words, hashtags or phrases to search for; the location where the resulting tweets will be saved; and whether we want to use the Search API or the Streaming API - the Search API goes back in time and the Streaming API goes forward, starting at execution time and continuing until the application is stopped. When using the Search API, the application returns tweets in batches. The length of each batch depends on the number of tweets set as a parameter in the GUI and the API window allowance. The search API returns up to 100 tweets per search, and permits 720 search requests per hour, giving us a maximum of 72,000 tweets per hour [23]. The search API can be used to retrieve tweets posted in the past few days. When using the Streaming API, a stream listener returns tweets when they are published. The Streaming API can collect all the tweets that contain up to 400 keyword phrases, were sent by up to 5,000 accounts, and originated in up to 25 geographic areas. Presumably, the Streaming API can retrieve up to $1 \%$ of the full firehose of tweets [23]. However, the total flow has not been documented.

As tweets are retrieved, they are saved on a MongoDB database. Some metadata associated with the tweets is stored together with the text of the tweets. The precise information that we store is listed below:

- tweet Text: The text of the tweet.

- tweetDate: The date when the tweet was publishedsecond and minute granularity are included in the date.

- simplifiedDate: The tweetDate reduced down to day granularity-i.e. 'dd-MM-yyyy'.

- tweetPolarity: The overall sentiment expressed in the tweet Text. The possibilities are positive, negative, neutral and balanced.

- overallsentiment: A number that denotes how positive or negative the tweet Text is.

- sentimentFound: A comma separated list of matching sentiment words present in the tweet Text.

The first two pieces of information-tweet Text and tweetDate-are actually retrieved from Twitter. The remaining information is calculated from the first two.

Extracting the sentiment expressed in the tweets returned from both the Search and Streaming API is at the core of our work. To determine the overall sentiment of a tweet, we loop through all the words in the tweet text and compare them to the sentiment words. If we encounter a sentiment word in the text of the tweet that is in the positive list, the overall sentiment value of the tweet is incremented by 1 ; if we find in the text of the tweet a word that is in the negative list, the overall sentiment value of the tweet is decremented by 1 . Prior to looping through the words of the tweet, such words are converted to lower case to prevent any case-sensitive mismatching. In summary, the sentiment analysis is performed in two parts: first, we identify the sentiment words; and then we determine the orientation, or polarity, of the tweet-i.e., whether the tweet is positive, negative, neutral or balanced.

If the overall sentiment is:

- Greater than 0 , the polarity is positive.

- Less than 0 , the polarity is negative. 
- Equal to 0, with no sentiment words present, the polarity is neutral.

- $\quad$ Equal to 0, with sentiment words present, the polarity is balanced.

The overall sentiment is calculated as follows:

- By default, the overall sentiment of all tweets is initially 0 .

- If a positive sentiment word is found, the overall sentiment is incremented by 1 .

- If a negative sentiment is found, the overall sentiment is decremented by 1 .

- After all the sentiment words have been checked against the tweet, a polarity is assigned to the tweet.

\section{RESULTS}

We have tested our approach using different examples in the political domain: Brexit, Trump, French Presidential Election and UK General Election. In this paper, we consider only UK General Election.

Table 1 shows the dataset composition. All of the data that is stored on the cloud can be used for analytics. As matches for the 6,800 sentiment words are found within the tweet, these words are recorded and stored.

TABLE I. DATASET COMPOSITION

\begin{tabular}{|c|c|}
\hline Number of records & $\mathbf{4 0 , 9 9 7}$ \\
\hline Hashtags/ words used & \#UKGeneralElection \\
& \#ge17 \\
& \#GeneralElection2017 \\
& \#Election2017 \\
& \#ge2017 \\
\hline
\end{tabular}

From looking at Figure 4, the UK election is a great candidate for this analytical process. The activity on this area was consistent with key events with the population being very opinionated on this area. As days progressed for the UK election, interest and activity on this topic was sustained. This can be seen in the consistently high spikes in the number of tweets retrieved. Sentimentally, the number of positive and negative tweets remained very close. This was expected due to the close results of the election when the results of the election are considered. Although there was consistently a high number of neutral tweets versus opinions, this is to be expected from almost all topic areas chosen. This is due to the fact that a lot of tweets do not always portray an emotion, some may be statements, observation, questions etc. In stating this, there was also a large percentage of tweets which did portray emotions. Observing Figure 4, which is showing sentiment change over time, it can be seen that on any given day the majority of sentiment shown is neutral, meaning there is no sentiment being displayed in the tweets. In seeing spikes in the number of neutral opinions (usually including a raise in positive and negative) it can be seen that interest/ activity in the topic area increases on those days. This correlates to significant events in the topic area occurring on those dates which are highlighted in the plot. Changes in the overall public feelings towards these significant events can be seen in the fluctuation of the number of positive and negative tweets being posted.

In order to compare the results obtained through the number of tweets received on the topic area, we used the global search results of Google Trends [12]. Google Trends considers interest over time: the numbers represent the search interest relative to the highest point on the chart for the given region and time. A value of 100 is the peak popularity for the term; a value of 50 means that the term is half as popular; a score of zero means the term was less than $1 \%$ as popular as the peak. For illustration purposes, the graph in Figure 5 uses the logarithm of the Interest over time, because we are studying a variable that is a function of a very large set of ordered magnitudes. In particular, we want to highlight the relative variations of the variable instead of the absolute ones.

In Figure 6, we present the same data as in Figure 4, but using the logarithm of the Number of tweets to enhance the relative variations. Figure 7 shows a comparison against the results of a re-elaboration of the poll tracker used by $\mathrm{BBC}$ News [24]; in such plot, different polling companies are used.

We also used word clouds [25] to visualize the words included in the tweets that we collected and explain their relevance to the events considered. The idea was to filter the tweets down to the words that appear with highest frequency. Prior to the creation of the word clouds, we removed the stop words from the tweets-i.e., we removed words that are extremely common and semantically non-selective, such as the, is, at and on. We employed the stop-word list built by Salton and Buckley for the experimental SMART information retrieval system [26], which contains 571 words. By removing these words, we guarantee that the word clouds are not cluttered with non-relevant text. The actual rendering of the word clouds was done with the help of WordClouds.com [27], a freely-available online tool. We looked into the main events of the campaigning period starting around $18^{\text {th }}$ April 2017. We computed the sentiment analysis of the tweets collected when the main events of the campaigning period took place.

The $18^{\text {th }}$ April 2017 T. May announced the plans election

The word clouds of Figure 1 A) shows the first effects of the announced plans election referencing words related to the vote in June, the importance of the first polls and the first campaigning themes like the Leave from UE and cuts tax.

We can note a peak of interest in Figure 4 considering the neutral sentiment and in Figure 5 through the Interest. In Figure 4 we can see in the close days after this event, an increment of the total number of positive and negative sentiments in the tweets. The relative trend, in Figure 6, is a reduction of the positive sentiment after the $18^{\text {th }}$ April.

The $4^{\text {th }}$ May 2017 the local election take place

The word clouds of Figure $1 \mathrm{~B}$ ) shows the importance of the vote in the local election and a continues referencing of website in the tweets ( $h t t p)$. The main parties like tory (conservatory), labour (labourist), ukip are often mentioned; also some important persons, like the leader of the Labour Corbyn and the foreign minister Boris, are considered.

In this date there is an interesting activity in terms of neutral, positive and negative sentiment in the tweets in Figure 4, with a relative peak of interest in Figure 5. It is interesting 

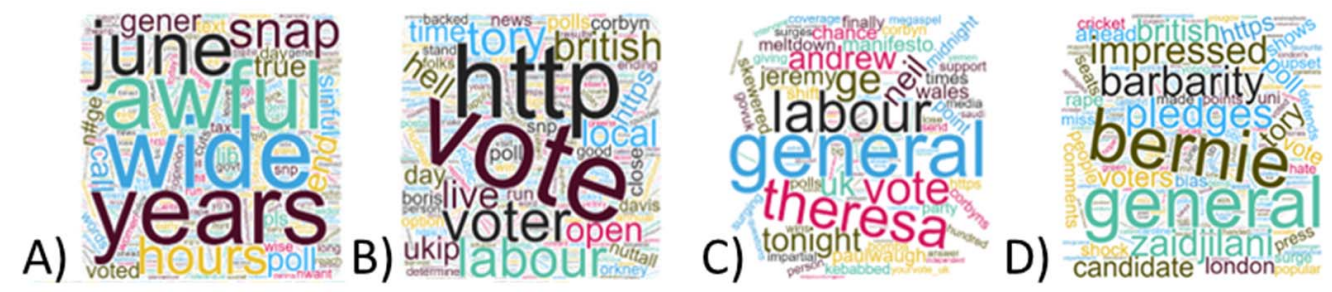

Fig 1. A) $18^{\text {th }}$ April 2017 Prime Minister May announced the plans election, B) $4^{\text {th }}$ May 2017 the local election take place, C) $22^{\text {nd }}$ May 2017 the campaign goes ahead with different topics and there is the Manchester terrorist attack, D) $2^{\text {nd }}$ June 2017 poll tracker shows Labour at highest level in almost three years
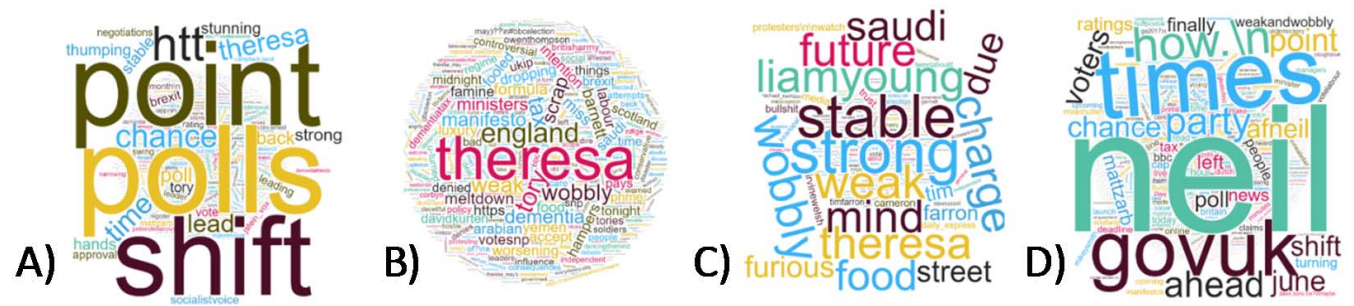

Fig. 2. $22^{\text {nd }}$ May 2017 cloud words based on the sentiment extracted from the tweets with our method: A) positive, B) negative, C) balanced, D) neutral.
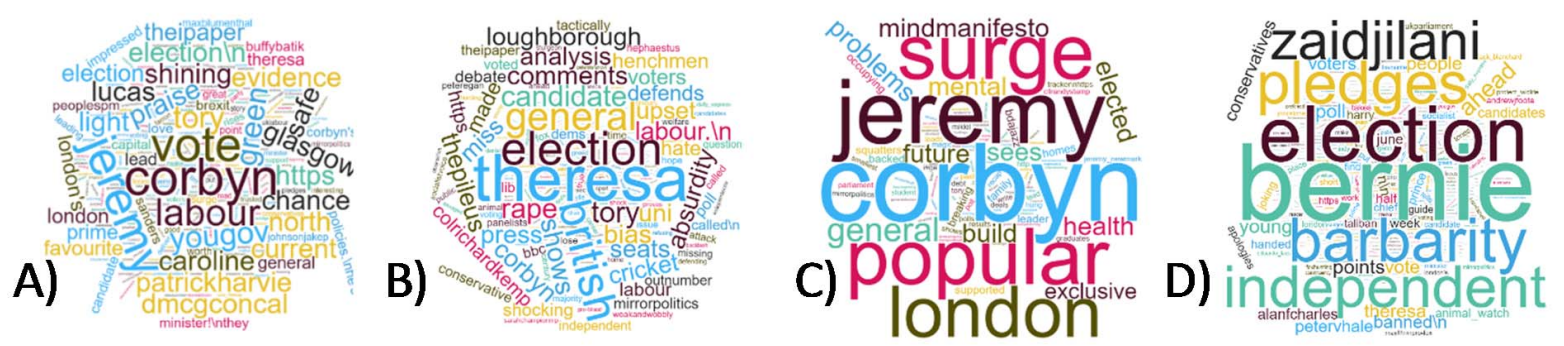

Fig. 3. $2^{\text {nd }}$ June 2017 cloud words based on the sentiment extracted from the tweets with our method: A) positive, B) negative, C) balanced, D) neutral.

to note in Figure 6 the continuous relative rise of the positive interest, before the election, followed by another rise of the positive sentiments after the results of the local elections.

The $22^{\text {nd }}$ May 2017 the campaigning go ahead with different topics and there is the Manchester terrorist attack.

The Manchester terrorist attack is considered in the tweets with several evidences in the cloud words of Figure 8, considering words like yemen, saudi, bombs, tonight; the Prime Minister Theresa May is considered the reference for the conservatory (there are no evidence of the word tory) against the labour and Corbyn. In addition, the party "uikp", very important during the Brexit "Leave" campaigning in the 2016, is not present in the tweets.

Comparing the positive sentiment of Figure $2 \mathrm{~A}$ ) with the negative sentiment of Figure $2 \mathrm{~B}$ ) it is possible to note that the Prime Minister Theresa May is the key of this election, which polarize the attention with an important negative relevance with respect to the campaigning themes and main parties. In the positive sentiment of $2 \mathrm{~A}$ ) there is the reference to the polls and shift. The words related to the Manchester attack produce negative sentiments in 2 B). The Figure 2 C) and D) show the difference between the balanced and the neutral sentiments.

There is a relative peak of people attention through the neutral sentiment in Figure 4, which is confirmed in Figure 5 considering the Interest. The positive sentiment has a peak bigger than the negative one in Figure 4, which corresponds a relative decreasing of the negative sentiment, the day after the event in the Figure 6. Considering the previous word clouds, it seems that the negative effect of the terrorist attack is less important than the positive effect of the recent polls.

The 2nd June 2017 poll tracker shows Labour at highest level in almost three years

In the cloud words of Figure 1 D) it is possible to note that while the UK campaigning maintains interest on pledges, parties (tory), candidate and pools, the news from US and Middle East are influencing the tweets in particular considering the Iran sanctions, with comments, shock, impressed and barbarity words. In particular these tweets are influenced by the news published in the Intercept report of Zaid Jilani (zaidjilani), and the important related comments from the American politician Bernie Sanders. 


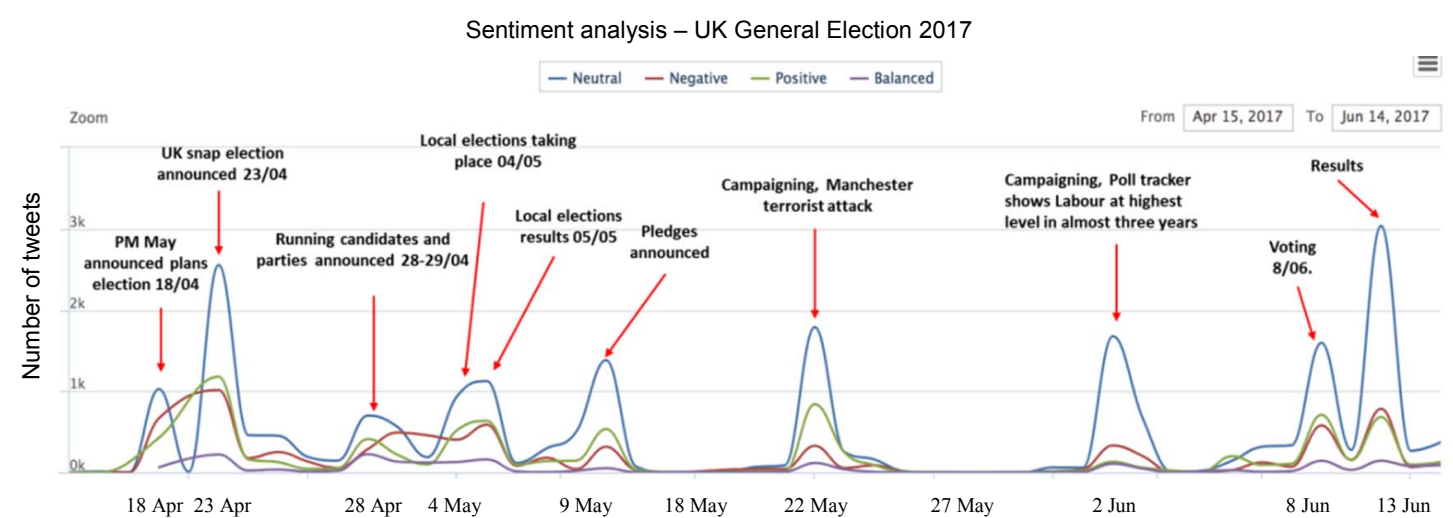

Fig.4 Twitter sentiment analysis, for the UK general Election 2017

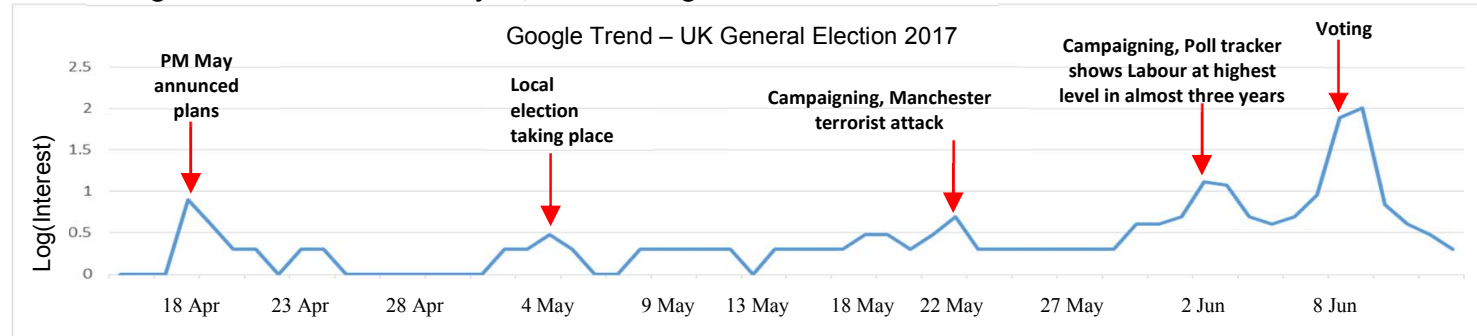

Fig.5 Re-elaboration of Google trends, using the log of the interests for the UK general Election 2017

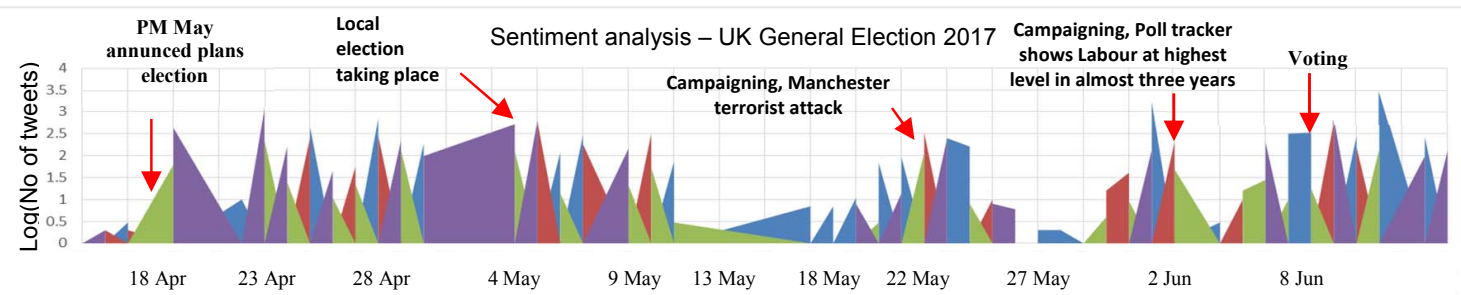

Fig. 6 Twitter sentiment analysis using the log of the number of tweets, for the UK general Election 2017

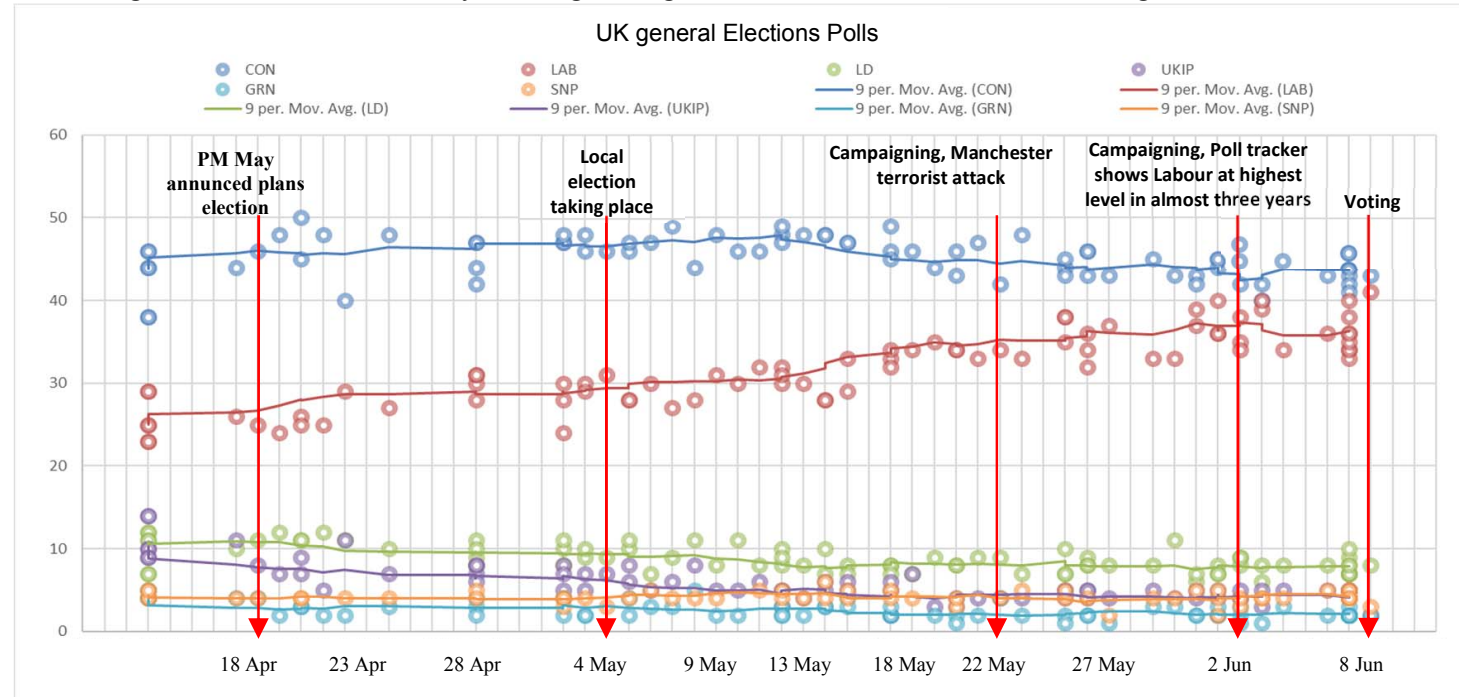

Fig. 7 Re-elaboration of the poll tracker, used by BBC NEWS, for the UK general Election 2017 
In Figure 3 we show in A) and B) the comparison of the positive sentiment among the negative. In the general positive sentiment, we can note several link to Labour like Corbyn, Jeremy, labour but also interest for the new position of Patrich Harvie (patrickharvie), in Glasgow, about the Scottish Green to have a tactical voting to block Conservatives (tory). In the word clouds of negative sentiment Theresa May and general election are the most important words. In Figure $3 \mathrm{C}$ ) also in the balanced sentiment Corbyn, Jeremy, and the labour manifesto and related topics (health, occupying, future, problems) have high interest. There is a relative peak of interest, in Figure 5, which correspond a peak of neutral sentiment in Figure 4. There is also a small peak related to the number of negative sentiment, in Figure 4, confirmed in Figure 6 with a relative max of negative sentiment. Such negative sentiments it seems to be correlated with Theresa May, in the previous discussion through the word clouds. In Figure 7 is reported the corresponding point of interest in the poll tracker.

\section{CONCLUSIONS}

We have presented a fully functional sentiment analysis tool for Twitter. The analytics carried out provide some value in relation to the overall public's feeling towards the specified topic area.

We believe that the strong correlation between the number of neutral tweets published over time and Google's search volume validates the use of our tool to timely monitor critical events in political campaigns. Indeed, whenever Google Trends identified a peak in the relative volume of searches made about the recent General Election, we also encountered an increase in the retrieval of neutral tweets.

This project provides a base for further development in this area. Opportunities for future work may involve:

- $\quad$ Taking into account the context of the tweets.

- Using geographical location to refine analysis.

- Using more hashtags to follow an event, like the elections. Furthermore, it could be interesting to study the correlation between another General Election and other specific topics related to the candidates.

- In the following data analysis related to political events, we will consider the effect of fake news and other important events that are external to the election campaigning.

- Exploring the possibility of aggregating data from different sources.

\section{REFERENCES}

[1] Jansen, B.J., et al., Twitter power: Tweets as electronic word of mouth Journal of the Association for Information Science and Technology, 2009. 60(11): p. 2169-2188.

[2] Twitter. Twitter. It's what's happening. 2017 [cited 2017; Available from: https://twitter.com/.

[3] InternetLiveStats.com. Twitter Usage Statistics. 2017 [cited 2017; Available from: http://www.internetlivestats.com/twitter-statistics/.

[4] Gueorguieva, V., Voters, MySpace, and YouTube. Social Science Computer Review, 2007. 26(3): p. 288-300.

[5] Širinic, D., et al. The International Conference on the Advances in Computational Analysis of Political Text. in PolText 2016. 2016. Dubrovnik, Croatia: University of Zagreb, Faculty of Political Science.
[6] Wang, Y., et al. To Follow or Not to Follow: Analyzing the Growth Patterns of the Trumpists on Twitter. in The 10th International AAAI Conference on Web and Social Media (ICWSM). 2016. Cologne, Germany.

[7] Wang, Y., Y. Li, and J. Luo. Deciphering the 2016 US Presidential Campaign in the Twitter Sphere: A Comparison of the Trumpists and Clintonists. in The International Conference On Web And Social Media. 2016. Cologne, Germany.

[8] Metaxas, P.T., E. Mustafaraj, and D. Gayo-Avello. How (not) to predict elections. in Privacy, Security, Risk and Trust (PASSAT) and 2011 IEEE Third Inernational Conference on Social Computing (SocialCom), 2011 IEEE Third International Conference on. 2011. IEEE.

[9] Jungherr, A., P. Jürgens, and H. Schoen, Why the Pirate Party Won the German Election of 2009 or The Trouble With Predictions: A Response to Tumasjan, A., Sprenger, T. O., Sander, P. G., \& Welpe, I. M. "Predicting Elections With Twitter: What 140 Characters Reveal About Political Sentiment". Soc. Sci. Comput. Rev., 2012. 30(2): p. 229-234.

[10] Ceron, A., et al., Every tweet counts? How sentiment analysis of social media can improve our knowledge of citizens' political preferences with an application to Italy and France. New Media \& Society, 2014. 16(2): p. 340-358.

[11] Liu, B., Sentiment analysis and opinion mining. Synthesis lectures on human language technologies, 2012. 5(1): p. 1-167.

[12] Google Inc. Google Trends. 2017 2017]; Available from: https://trends.google.co.uk/.

[13] Tumasjan, A., et al. Predicting elections with twitter: What 140 characters reveal about political sentiment. in The International AAAI Conference on Web and Social Media (ICWSM). 2010. Washington, DC.

[14] O'Connor, B., et al. From tweets to polls: Linking text sentiment to public opinion time series. in The International Conference on Weblogs and Social Media. 2010. Washington, DC.

[15] Asur, S. and B.A. Huberman. Predicting the future with social media. in IEEE/WIC/ACM International Conference on Web Intelligence and Intelligent Agent Technology (WI-IAT). 2010. Toronto, Canada: IEEE.

[16] Twitter, I. Twitter Developer Documentation - API Overview. 2017 [cited 2017; Available from: https://dev.twitter.com/overview/api.

[17] JetBrains. IntelliJ IDEA: The Java IDE for Professional Developers. 2017 [cited 2017; Available from: https://www.jetbrains.com/idea/.

[18] Yamamoto, Y. Twitter4J. 2017 [cited 2017; Available from: http://twitter4j.org/en/.

[19] MongoDB, I. MongoDB Atlas. 2017 Available from: $\mathrm{https}: / / \mathrm{www} . \mathrm{mongodb} . \mathrm{com} / \mathrm{cloud} /$ atlas.

[20] MongoDB, I. MongoDB Compass. 2017 [cited 2017; Available from: https://www.mongodb.com/products/compass.

[21] Hu, M. and B. Liu. Mining and summarizing customer reviews. in Proceedings of the ACM SIGKDD International Conference on Knowledge Discovery and Data Mining. 2004. Seattle, WA: ACM.

[22] Knowi. Adaptive Intelligence for Modern Data. 2017 [cited 2017; Available from: https://knowi.com/.

[23] 140 Dev LLC. Aggregating tweets: Search API vs. Streaming API. Twitter API Programming Tips, Tutorials, Source Code Libraries and Consulting 2013 [cited 2017; Available from: http://140dev.com/twitter-api-programming-tutorials/aggregatingtweets-search-api-vs-streaming-api/.

[24] BBC News. Election 2017 poll tracker: Opinion poll data. 20177 June 2017 [cited 2017; Available from: http://www.bbc.co.uk/news/election2017-39869459.

[25] Heimerl, F., et al. Word cloud explorer: Text analytics based on word clouds. in System Sciences (HICSS), 2014 47th Hawaii International Conference on. 2014. IEEE.

[26] Salton, G., The SMART retrieval system: Experiments in automatic document processing. IEEE Transactions on Professional Communication. Vol. PC-15. 1972, Englewood Cliffs, NJ: PrenticeHall. 17-17.

[27] Zygomatic. WordClouds.com. 2017 [cited 2017; Available from: http://www.wordclouds.com/. 